Proc. of the 15th Int. Workshop on Slow Positron Beam Techniques and Applications, Prague, September 2-6, 2019

\title{
Studies of Ortho-Positronium Decays into Three Photons with the J-PET Detector
}

\author{
A. Gajos* on Behalf of the J-PET COllaboration \\ Institute of Physics, Jagiellonian University, S. Łojasiewicza 11, 30-348 Cracow, Poland
}

\begin{abstract}
The Jagiellonian Positron Emission Tomograph (J-PET) setup, besides being the first PET scanner built with plastic scintillators is currently used to conduct a broad range of experiments involving ortho-positronium (o-Ps) decays into three photons. We present results of studies of o-Ps $\rightarrow 3 \gamma$ decays performed in J-PET with a view to search for angular correlations between the photons' momenta and positronium spin direction which would violate the combined CPT symmetry, scarcely tested in leptonic systems. To date, the most precise CPT test using ortho-positronium decays reached the precision of $3 \times 10^{-3}$ whereas effects limiting the sensitivity are only expected at the level of $10^{-9}$. In the discussed J-PET measurement, ortho-positronium atoms are created by positrons from a ${ }^{22} \mathrm{Na}$ source thermalizing in an extensive-size cylindrical target of mesoporous silica and decay positions are reconstructed using a trilateration-based technique. Decay photons are recorded by 192 strips of plastic scintillators with high timing resolution. Such a setup allows for registration of an unprecedented spectrum of geometrical configurations of o-Ps $\rightarrow 3 \gamma$ decays including also correlations with positronium spin. With an angular resolution and o-Ps polarization control improved with respect to previous measurements, J-PET aims at achieving the sensitivity of CPT test at a precision level of at least $10^{-4}$.
\end{abstract}

DOI: 10.12693/APhysPolA.137.126

PACS/topics: 11.30.Er, 36.10.Dr, 13.40.Hq

\section{Introduction}

Although extensive experimental tests of fundamental discrete symmetries have been performed in the last decades, most of the experiments concentrated on systems constituted by quarks, with relatively little emphasis on the leptonic sector. Among purely leptonic systems, the two viable candidates for discrete symmetry studies are measurements of neutrino oscillations and decays of positronium atoms. While the former has already started to deliver an insight into the CP-violating Dirac phase through long-baseline neutrino experiments $[1,2]$, exploiting an alternative system is desirable.

The most precise test of the CPT symmetry using ortho-positronium (o-Ps) annihilations has reached the precision of $3 \times 10^{-3}$ (yielding a null result) while the physical limitations on the achievable precision should only arise at the $10^{-9}$ level due to $\gamma \gamma$ interactions in the final state [3]. Therefore, the J-PET collaboration aims at testing CPT through search for prohibited angular correlations in o-Ps $\rightarrow 3 \gamma$ with a precision improved by at least an order of magnitude. In this work, we present the first studies of o-Ps $\rightarrow 3 \gamma$ annihilations in a setup allowing for event-by-event positronium spin estimation in J-PET with a view to searching for CPT-odd angular correlations in this process.

\section{The J-PET detector}

The Jagiellonian Positron Emission (J-PET) detector was constructed as the first PET tomography device

\footnotetext{
*e-mail: aleksander.gajos@uj.edu.pl
}

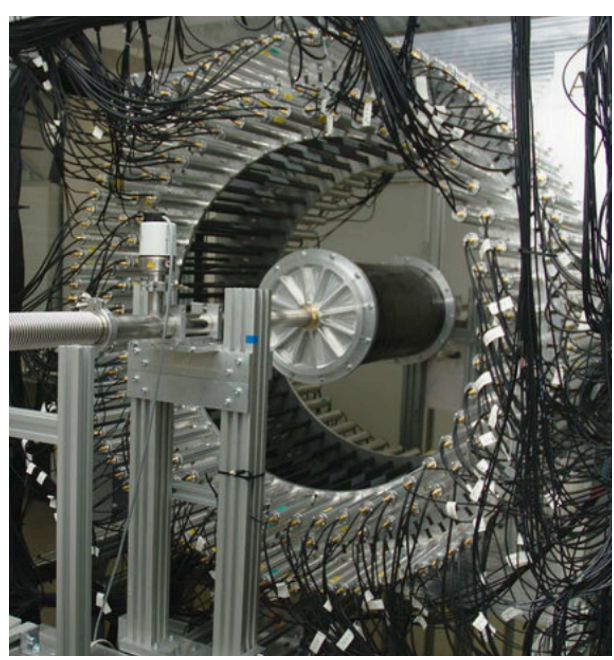

Fig. 1. The cylindrical vacuum chamber whose inner walls are coated with a porous medium for enhanced o-Ps production, mounted along the axis of the J-PET detector.

based on plastic scintillators [4]. However, it is a robust general-purpose photon detector capable of recording $\mathrm{o}-\mathrm{Ps} \rightarrow 3 \gamma$ annihilations in an inclusive measurement with a large geometrical acceptance. J-PET is constituted by three concentric cylindrical layers of axially-arranged plastic scintillator strips of $50 \mathrm{~cm}$ length, each read out at both ends by vacuum tube photomultipliers (see Fig. 1).

Longitudinal position and time of $\gamma$ interactions in the scintillator strips are reconstructed using times of light propagation from the point of $\gamma$ Compton scattering to the ends of the strip, providing interaction time resolution at the level of about 100 ps thanks to the fast signals of plastic scintillators $[5,6]$. 


\section{Production and spin control of o-Ps}

The effects of violation of the CPT symmetry can be sought in the annihilations of o-Ps atoms into three photons through an asymmetry in the $\boldsymbol{S} \cdot\left(\boldsymbol{k}_{1} \times \boldsymbol{k}_{2}\right) /\left|\boldsymbol{k}_{1} \times \boldsymbol{k}_{2}\right|$ operator, where $\boldsymbol{S}$ is the o-Ps spin and $\boldsymbol{k}_{i}$ denote the momenta of emitted photons, labeled in the order of decreasing energy. Non-zero mean value of such operator, corresponding to a non-vanishing angular correlation between the positronium spin and the annihilation plane, would be a sign of CPT non-conservation [3, 7].

Evaluation of this operator requires knowledge of the o-Ps spin which can be obtained either by using a polarized positron beam to produce the positronia [8], polarizing the o-Ps sample along a given quantization axis using external magnetic field (as done e.g., in a test of the CP symmetry using a similar principle described in Ref. [9]) or by relying on the average polarization of positrons from a $\beta^{+}$decay along their direction of emission to a degree of $P_{\mathrm{e}+}=\bar{v} / c$ where $\bar{v}$ is the average positron speed. The latter approach was exploited in the most precise test of the CPT symmetry with o-Ps performed to date, performed using the Gammasphere detector [10], where positrons from a $\beta^{+}$source were allowed to form positronia in an aerogel hemisphere around it, which effectively limited their emission directions to a $2 \pi$ solid angle. Therefore, the effective polarization of the produced ortho-positronia was given by

$$
P_{\mathrm{o}-\mathrm{Ps}}=\frac{2}{3} P_{\mathrm{e}+} \approx \frac{2}{3} \frac{v}{c} \frac{1}{3}(1+\cos \alpha),
$$

where the last factor results from the uncertainty of $\mathrm{e}^{+}$ emission direction being described by a cone with an opening angle $2 \alpha$ [11]. With the aerogel hemisphere target used in Gammasphere, this geometrical polarization limiting factor amounted to $(1+\cos (\pi / 2)) / 2=0.5[10]$.

In the J-PET experiment, we propose an extension of this approach significantly reducing this geometrical limitation on o-Ps sample polarization. The positronium production setup is schematically presented in Fig. 2. We use a cylindrical $(R=12 \mathrm{~cm})$ vacuum chamber with a small $\beta^{+}$source mounted in its center while the inner walls of the chamber are coated with a $3 \mathrm{~mm}$ layer of porous silica (R60G) enhancing positronium formation [12]. Thickness of the layer has been chosen to be well above the expected penetration depth of positrons from the ${ }^{22} \mathrm{Na}$ source [13].

A pressure of $10^{-4}$ mbar is maintained in the chamber to reduce scattering of positrons before they reach the porous medium. In order to reduce Compton scattering and absorption of photons in the sub-511 $\mathrm{keV}$ range in the external chamber walls, they are made of $3 \mathrm{~mm}$ thick polycarbonate [14]. Figure 1 presents the positronium production chamber mounted inside the J-PET detector.

When positrons reach the chamber wall, thermalize and form positronium in the porous material, the gamma quanta from an o-Ps $\rightarrow 3 \gamma$ annihilation can be recorded by J-PET in an exclusive measurement, allowing for reconstruction of the annihilation point using a novel

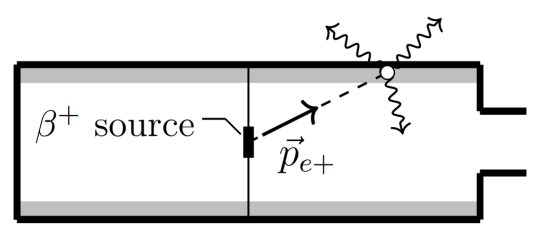

Fig. 2. Principle of the event-by-event estimation of the spin direction of positrons forming ortho-positronia. The $e^{+}$momentum direction (dashed line) is estimated using the location of the $\beta^{+}$source in the center of the positronium production chamber (thick black lines) and the reconstructed annihilation point of o-Ps (white dot) formed in the $3 \mathrm{~mm}$ layer of mesoporous silica covering the inner wall of the chamber (gray).

trilateration-based technique [15]. Consequently, knowledge of the latter provides an estimate of the positron emission direction as presented in Fig. 2. This allows for estimating the incident positron spin on an eventby-event basis where the geometrical factor from Eq. (1) comes mostly from the uncertainty of the o-Ps annihilation point, leading to $\alpha \approx 15^{\circ}$ and the geometrical polarization limitation factor of only 0.98 [15].

\section{Recording of o-Ps $\rightarrow 3 \gamma$ decays with J-PET}

The positronium production setup described in the previous section combined with a $10 \mathrm{MBq}{ }^{22} \mathrm{Na}$ source was used to record the o-Ps $\rightarrow 3 \gamma$ annihilations in a 60-day measurement. In order to minimize the event selection bias, the J-PET data were acquired in a triggerless mode. Analysis of the whole collected dataset is in progress at the moment of presentation of this work.

Usage of the sodium source entails presence of 1.27 MeV photons from deexcitation of $\mathrm{Ne}^{*}$ resulting from the $\beta^{+}$decay, which need to be disentangled from the annihilation photons. As the gamma quanta are detected in the plastic scintillators through their Compton scattering, the deposited energies come from continuous spectra limited from above by Compton edges dependent on the original incident photon energy. J-PET uses the time over threshold (TOT) technique applied to photomultiplier signals to provide a measure of the energy deposited by gamma quanta in the scintillators [16]. The TOT measurement allows for selection of two distinct TOT regions below and above the Compton edge for $511 \mathrm{keV}$ photons, respectively for identification of o-Ps annihilation and $\mathrm{Ne}^{*}$ deexcitation photons.

In order to recognize o-Ps $\rightarrow 3 \gamma$ events, a set of three annihilation photon candidates identified using TOT and recorded in distinct scintillator strips is required within a time window of $2.5 \mathrm{~ns}$. Independently, the photons classified as deexcitation quanta can be used to provide a start signal for measurement of the decay time of an o-Ps candidate event. Figure 3 presents the relative multiplicity of annihilation and deexcitation photon candidates as identified using TOT, recorded in a broad time window of $250 \mathrm{~ns}$ allowing to see the o-Ps lifetime. 


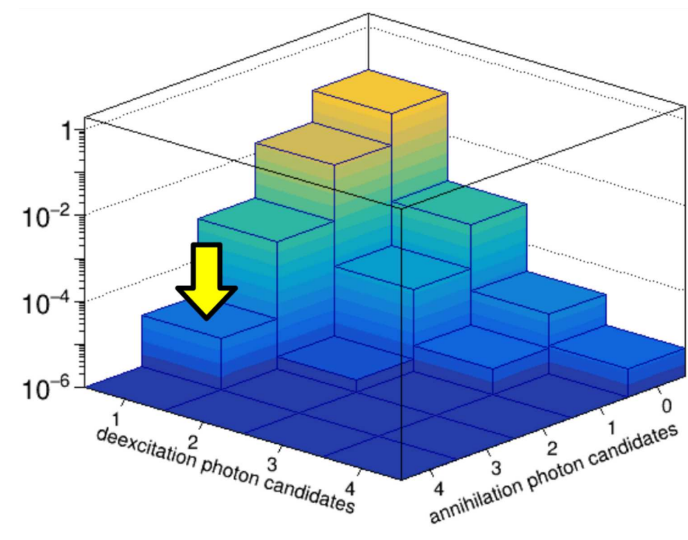

Fig. 3. Fractional multiplicities of the number of o-Ps annihilation candidate photons versus the number of deexcitation candidate photons from the $\beta^{+}$emitter, where the latter was observed within $250 \mathrm{~ns}$ before the annihilation time. The yellow arrow denotes the combination used for studies described in this work, constituted by three annihilation photon candidates and a single deexcitation candidate.

Fully recorded o-Ps $\rightarrow 3 \gamma$ event candidates with an associated prompt photon for decay time information constitute about $10^{-5}$ of all collected data. For all such event candidates, the point and time of the $3 \gamma$ annihilation is reconstructed using the trilaterative technique [15].

Subsequently, a set of criteria dedicated to identification of $\gamma \gamma$ back-to-back annihilations are applied in order to discriminate the background from direct $\mathrm{e}^{+} \mathrm{e}^{-}$ annihilations and para-positronium decays. As photons are recorded in J-PET through their Compton scattering in the plastic scintillators, an already-recorded primary photon can undergo a second scattering in a different detection module, creating a spurious recorded interaction which may be misinterpreted as another primary photon. This effect constitutes a dominant source of background after removal of $\gamma \gamma$ events but is largely eliminated by a dedicated criterion testing the time of flight hypothesis of a photon travelling directly between two recorded $\gamma$ interaction points.

Figure 4 presents the distribution of the transverse radius (including polar Jacobian weighting) of the reconstructed o-Ps $\rightarrow 3 \gamma$ event candidates surviving the selection described above. An enhancement in the region of the porous material location in the positronium production chamber (at $R=12 \mathrm{~cm}$ ) is clearly visible, indicating the presence of o-Ps annihilations in the J-PET data and proving the possibility to reconstruct the position of such annihilations taking place in an extensivesize medium. It should be noted that the demonstrated method of reconstructing o-Ps annihilations additionally enables studies of positronium decays in living organisms, opening new perspectives in medical diagnostics [17, 18]. Further procedures for purification of the event sample as well as for improving the decay point spatial resolution are presently under elaboration.

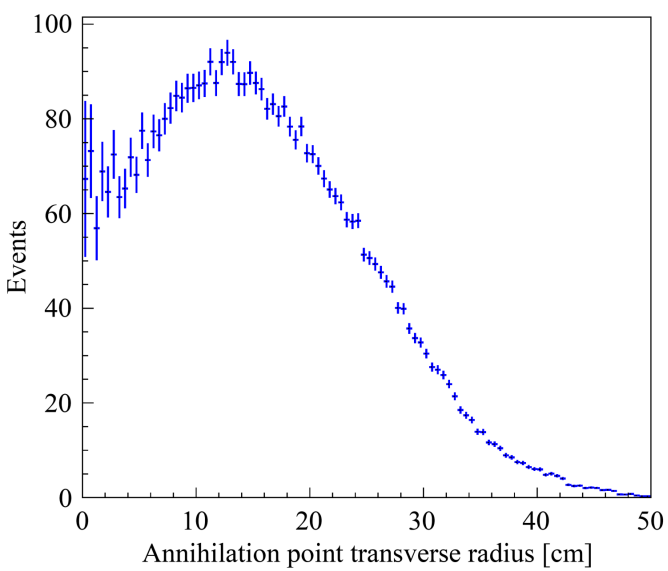

Fig. 4. Transverse radius of the reconstructed o-Ps $\rightarrow$ $3 \gamma$ annihilation points for event passing all selection criteria. The distribution includes weighting by the polar coordinates' Jacobian. Maximal number of events is observed around $R=12 \mathrm{~cm}$, in agreement with expectations based on the geometry of the positronium production medium used.

\section{Conclusions and perspectives}

The J-PET experiment studies the o-Ps $\rightarrow 3 \gamma$ annihilations with a view of measuring the mean value of the $\boldsymbol{S} \cdot\left(\boldsymbol{k}_{\mathbf{1}} \times \boldsymbol{k}_{2}\right) /\left|\boldsymbol{k}_{1} \times \boldsymbol{k}_{2}\right|$ CPT-asymmetric operator thus conducting a test of this symmetry. The analysis of the performed measurement demonstrates the capability of J-PET to produce ortho-positronia, identify their $3 \gamma$ annihilations and reconstruct their spatial positions in an extensive-size medium, allowing for an event-by-event positronium spin direction estimation. The latter offers a significant improvement of the control of o-Ps polarization with respect to the current best measurement. Moreover, J-PET is able to record a broad range of geometrical configurations of o-Ps $\rightarrow 3 \gamma$ events, allowing to minimize geometrical asymmetry contributions to the systematic uncertainties in the measurement. Finally, other factors such as angular and timing resolution would allow for a further reduction of systematic uncertainties with respect to the most precise result to date. In terms of statistical uncertainty, the dataset already collected (corresponding to about a month of continuous measurement) is expected to allow for reaching a sensitivity at the level of $10^{-4}$. Longer future measurements can be used to further increase the statistical sensitivity.

The long-term plans of J-PET feature adding a fourth layer of scintillator strips with silicon photomultipliers and fully digital readout to increase the geometrical acceptance and using a spherical positronium production chamber which would enhance the probability of positrons from the $\beta^{+}$source forming a positronium in the most sensitive region of the detector. With these improvements combined with increased statistics, J-PET should be able to search for the CPT-violating asymmetry at the precision level of $10^{-5}$. 


\section{Acknowledgments}

The J-PET collaboration acknowledges the support by the Polish National Center for Research and Development through grant INNOTECH-K1/IN1/64/ 159174/NCBR/12, the Foundation for Polish Science through the MPD and TEAM POIR.04.04.00-004204/17 programmes, the National Science Centre of Poland through grants no. 2016/21/B/ST2/01222 and $2017 / 25 / \mathrm{N} / \mathrm{NZ1} / 00861$ and the Ministry for Science and Higher Education through grants no. $6673 / \mathrm{IA} / \mathrm{SP} / 2016$ and $7150 / \mathrm{E}-338 / \mathrm{SPUB} / 2017 / 1$

\section{References}

[1] T2K Collaboration, Phys. Rev. Lett. 121, 171802 (2018).

[2] NOvA Collaboration, Phys. Rev. D98, 032012 (2018).

[3] W. Bernreuther, U. Low, J.P. Ma, O. Nachtmann, Z. Phys. C41, 143 (1988).

[4] S. Niedźwiecki, P. Białas, C. Curceanu, et al., J-PET Collaboration, Acta Phys. Pol. B 48, 1567 (2017).

[5] P. Moskal, Sz. Niedźwiecki, T. Bednarski, et al., Nucl. Instrum. Methods Phys. Res. A 764, 317 (2014).

[6] P. Moskal, N. Zoń, T. Bednarski, et al., Nucl. Instrum. Methods Phys. Res. A 775, 54 (2015).
[7] G.S. Adkins, in: Proc. 5th Meeting on CPT and Lorentz Symmetry (CPT 10), Bloomington (IN) 2010, p. 254.

[8] B.K. Arbic, S. Hatamian, M. Skalsey, J. Van Hause, W. Zheng, Phys. Rev. A 37, 3189 (1988).

[9] T. Yamazaki, T. Namba, S. Asai, T. Kobayashi, Phys. Rev. Lett. 104, 083401 (2010).

[10] P.A. Vetter, S.J. Freedman, Phys. Rev. Lett. 91 , 263401 (2003).

[11] P. Coleman, Positron Beams and Their Applications, World Sci., 2000.

[12] R. Ferragut, M. Dupasquier, A. Calloni, G. Consolati, F. Quasso, M.P. Petkov, S.M. Jones, A. Galarneau, F. Di Renzo, J. Phys. Conf. Ser. 262, 012020 (2011).

[13] J. Dryzek, Acta Phys. Pol. A 107, 598 (2005).

[14] M. Gorgol, B. Jasińska, M. Kosior, E. Stępień, P. Moskal, Acta Phys. Pol. B 51, 293 (2020).

[15] A. Gajos, D. Kamińska, E. Czerwiński, et al., Nucl. Instrum. Methods Phys. Res. A 819, 54 (2016).

[16] S. Sharma, EPJ Web Conf. 19905014 (2019).

[17] P. Moskal, B. Jasińska, E. Stępień, S.D. Bass, Nature Rev. Phys. 1, 527 (2019).

[18] P. Moskal, D. Kisielewska, C. Curceanu, et al., Phys. Med. Biol. 64, 05501 (2019). 